\title{
Dispersive crystal field excitations and quadrupolar interactions in $\mathrm{UPd}_{3}$
}

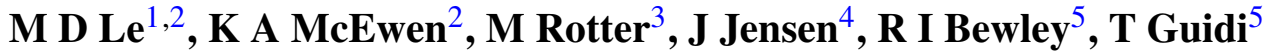 \\ and D Fort ${ }^{6}$ \\ ${ }^{1}$ Helmholtz-Zentrum Berlin, Hahn-Meitner-Platz 1, D-14109 Berlin, Germany \\ 2 Department of Physics and Astronomy, and London Centre for Nanotechnology, University College \\ London, London, WC1E 6BT, UK \\ ${ }^{3}$ Max Planck Institute for Chemical Physics of Solids, Nöthnitzer Straße 40, D-01187 Dresden, \\ Germany \\ ${ }^{4}$ Niels Bohr Institute, Universitetsparken 5, DK-2100 Copenhagen, Denmark \\ ${ }^{5}$ ISIS Facility, Rutherford Appleton Laboratory, Chilton, Didcot, OX11 0QX, UK \\ ${ }^{6}$ Department of Metallurgy and Materials, University of Birmingham, Birmingham, B15 2TT, UK
}

E-mail: duc.le@helmholtz-berlin.de

Received 18 August 2011, in final form 28 October 2011

Published 9 December 2011

Online at stacks.iop.org/JPhysCM/24/036002

\begin{abstract}
We report inelastic neutron scattering measurements and random phase approximation calculations of the dispersive crystal field excitations of $\mathrm{UPd}_{3}$. The measured spectra at lower energies agree with those calculated using quadrupolar interaction parameters deduced from bulk and x-ray scattering measurements. The more intense excitations arising from the hexagonal sites were used to obtain exchange parameters which proved to be anisotropic.
\end{abstract}

(Some figures may appear in colour only in the online journal)

\begin{abstract}
Measurements of dispersive electronic excitations by inelastic neutron scattering can yield detailed information about the interactions between electrons in a crystalline solid. The measured neutron scattering function $S(\mathbf{Q}, \omega)$ is proportional to the imaginary part of the dynamical susceptibility, $\chi^{\prime \prime}(\mathbf{Q}, \omega)$, which may be calculated given a knowledge of the inter-ion interactions using the random phase approximation [1]. In many magnetic compounds these interactions are the familiar exchange interactions between the spins of localized open-shell electrons on neighbouring ions, mediated by delocalized conduction electrons. In some rare earth systems, however, the larger orbital angular momentum of the electrons results in anisotropic charge or magnetic moment densities, from which strong multipolar interactions may arise. Such compounds may then undergo transitions to phases where these multipole moments are ordered.

$\mathrm{UPd}_{3}$ has been shown to exhibit quadrupolar order, with a series of four phase transitions below $7.8 \mathrm{~K}$, in which the electric quadrupolar moments of localized $5 \mathrm{f}$ electrons on neighbouring $U$ ions align in antiphase order along the crystallographic a direction [2]. The x-ray resonant scattering (XRS) measurements which demonstrated this quadrupolar

ordering suggested that the ordering occurs only between electrons on one of the two crystallographically inequivalent $\mathrm{U}^{4+}$ sites, namely sites with $\mathrm{D}_{3 \mathrm{~d}}(\overline{3} \mathrm{~m})$ point symmetry, which we shall call the quasi-cubic sites. Electrons on the other uranium site of the double hexagonal close packed (dhcp) structure (space group $P 6_{3} / m m c$ ), with hexagonal $\mathrm{D}_{3 \mathrm{~h}}(\overline{6} \mathrm{~m} 2)$ symmetry, appear not to show long range order. Nonetheless the excitations of electrons on these sites are important because they have much higher inelastic neutron scattering cross-sections than similar crystal field excitations of electrons on the quasi-cubic sites, due to the larger dipole matrix element of the hexagonal site transitions.

They are thus more clearly seen in our measurements, which were made using the MERLIN [3] neutron time-offlight spectrometer at ISIS on a $17 \mathrm{~g}$ single crystal of $\mathrm{UPd}_{3}$ grown by the Czochralski method. The spectrometer was operated mainly at incident energies of 25 and $16 \mathrm{meV}$ using the 'sloppy' chopper from MARI, as dedicated choppers for MERLIN were not yet ready at the time of the experiment. The choppers were rotated at $200 \mathrm{~Hz}(150 \mathrm{~Hz})$ for $E_{\mathrm{i}}=$ $25 \mathrm{meV}\left(E_{\mathrm{i}}=16 \mathrm{meV}\right)$ giving energy resolutions at the elastic line of approximately $1.2 \mathrm{meV}(0.8 \mathrm{meV})$.
\end{abstract}


The crystal was mounted in an Orange cryostat and cooled to $3.5 \mathrm{~K}$, below the lowest temperature quadrupolar phase transition. It was oriented with the $\mathbf{a}^{\star}$ and $\mathbf{c}^{\star}$ directions in the horizontal scattering plane, and such that the $\mathbf{c}^{\star}$ direction was initially parallel to the incident neutron beam. The crystal was then rotated in $1^{\circ}$ steps over $90^{\circ}$ until the $\mathbf{a}^{\star}$ direction was parallel to $\mathbf{k}_{i}$. At each step, a short 20 min exposure was taken, and these were subsequently combined using the Horace program [4] to construct the 4D $S(\mathbf{Q}, \omega)$, from which cuts in arbitrary constant $Q$-directions may be obtained. Since the 2D detector array and neutron time-of-flight allow only a mapping of a 3D surface in $\mathbf{Q}-\omega$ space, an additional degree of freedom, the sample rotation, is required to determine the full $4 \mathrm{D} S(\mathbf{Q}, \omega)$. This $4 \mathrm{D}$ reconstruction is desirable because the $3 \mathrm{D}$ surface in reciprocal space measured at a given crystal orientation is fixed by the geometry of the detectors and may not include all wavevector transfers $\mathbf{Q}$ which are of interest. Furthermore, if the crystal rotation is small enough, there are large overlaps in the $(\mathbf{Q}, \omega)$-space between each scan, so that although individual scans are of short duration, and thus have relatively poor statistics, the reconstructed data will have comparable statistics to a much longer traditional scan.

A background measurement of the empty cryostat was subtracted from the data, which were then folded along the principal symmetry axes to improve statistics. The top panels of figure 1 show a 2D slice of the reconstructed $4 \mathrm{D} S(\mathbf{Q}, \omega)$ at $25 \mathrm{meV}$ incident energy along the $\mathbf{a}^{\star}$ direction. The dispersive hexagonal site crystal field excitation centred around $16 \mathrm{meV}$ is clearly visible. There is also evidence in the data for the quasi-cubic site excitation centred around $10 \mathrm{meV}$, but the dispersion is hard to discern from the slice. Instead, the dispersion of the quasi-cubic excitations was determined from fitting Gaussian peak shapes to 1D cuts of the data. The better resolution data using $16 \mathrm{meV}$ incident energy were mainly used for this purpose, giving the dispersion shown in figure 2.

The crystal field splitting of the hexagonal site $\mathrm{U}^{4+}$ ions is particularly simple because the low lying energy levels are a $\Gamma_{1}$ singlet $\left(|s\rangle=\left|J=4, J_{z}=0\right\rangle\right)$ ground state and excited $\Gamma_{2}$ doublet $\left(|d\rangle=\left|J=4, J_{z}= \pm 1\right\rangle\right)$ at $\Delta \sim 16 \mathrm{meV}$ [6]. Because of the wavefunctions, the only allowed transitions on the hexagonal sites from the ground state singlet are to this $16 \mathrm{meV}$ doublet. Furthermore, inelastic neutron measurements on powder samples at higher temperatures [7, 8] indicate that there are no other levels below $16 \mathrm{meV}$. Therefore, the excited levels would only become thermally occupied at $\approx 150 \mathrm{~K}$ which is much higher than the measurement temperature of $3.5 \mathrm{~K}$, so that we need consider only transitions from the ground state.

This singlet-doublet or XY model has been considered within the RPA [1], under which the dynamical susceptibility is

$$
\chi_{\alpha \alpha}(\mathbf{q}, \omega)=\frac{2 n_{\mathrm{sd}}\left|\left\langle s\left|\hat{J}_{\alpha}\right| d\right\rangle\right|^{2} \Delta}{E_{q}^{2}-(\hbar \omega)^{2}}
$$

where $\alpha$ labels the directions $x$ and $y, n_{\mathrm{sd}}$ is the difference in thermal occupation between the singlet and doublet, and $E_{q}$ is the dispersion relation

$$
E_{q}=\left[\Delta\left(\Delta-2 n_{\mathrm{sd}}\left|\left\langle s\left|\hat{J}_{\alpha}\right| d\right\rangle\right|^{2} \mathcal{J}_{\alpha \alpha}(\mathbf{q})\right)\right]^{1 / 2}
$$

where $\mathcal{J}_{\alpha \alpha}$ is the exchange parameter. As the excited state is a doublet, one expects two excitations per ion in the unit cell. For an isotropic interaction, where $\mathcal{J}_{x x}=\mathcal{J}_{y y}$, there will be two doubly degenerate modes rather than four, because the coupling to each level of the doublet will be the same. This sufficed to explain the triple-axis spectrometer data measured by Buyers et al [9], where only two modes were observed. However, their model does not fit our data over a larger range in wavevector transfer, where for example near (102) in figure 1 we observed evidence of three modes, as seen in the intensity plot of the data in the top row. Unfortunately, the one-dimensional cuts of the data used to fit the peak positions shown in the bottom row had to be integrated over a relatively large range in $l$ to obtain enough neutron counts for a fit. This meant that the two lower modes become unresolved, so that only a single peak can be fitted. However, further features of the observed dispersion, such as the apparent mode crossing at (102) and (003) are best explained by the presence of more than two non-degenerate modes.

Therefore, we have used a similar model to [9] but with anisotropic exchange, which nonetheless obeys the symmetry of the $P 6_{3} / m m c$ space group [10]. This means that at high symmetry points in the Brillouin zone, such as the $\Gamma$, A and $\mathrm{K}$ points with six-fold symmetry, modes arising from the same single-ion transitions are degenerate. The Hamiltonian

$$
\begin{aligned}
\mathcal{H}= & \sum_{i} \mathcal{H}_{\mathrm{cf}}(i)-\frac{1}{2} \sum_{i j}\left\{\mathcal{J}_{1}^{i j}\left[\hat{J}_{x}^{i} \hat{J}_{x}^{j}+\hat{J}_{y}^{i} \hat{J}_{y}^{j}\right]\right. \\
& +\mathcal{K}_{1}^{i j}\left[\cos \left(2 \phi_{i j}\right)\left(\hat{J}_{x}^{i} \hat{\jmath}_{x}^{j}-\hat{J}_{y}^{i} \hat{J}_{y}^{j}\right)\right. \\
& \left.\left.+\sin \left(2 \phi_{i j}\right)\left(\hat{J}_{x}^{i} \hat{\jmath}_{y}^{j}+\hat{J}_{y}^{i} \hat{J}_{x}^{j}\right)\right]\right\}
\end{aligned}
$$

is obtained by adding an anisotropic exchange term with parameter $\mathcal{K}^{i j}$ in addition to the isotropic exchange with parameter $\mathcal{J}^{i j}$. The details of the crystal field Hamiltonian, $\mathcal{H}_{\mathrm{cf}}(i)$, for the $i$ th ion are given in [8] but are not important for the model, except to determine the single-ion energy $\Delta=15.7 \mathrm{meV}$ of the doublet levels. The anisotropic term was derived by considering the symmetry allowed interaction between pairs of ions, $i$ and $j$, expressed in a local coordinate system where the $x^{\prime}$ axis is the projection of $\mathbf{r}_{i}-\mathbf{r}_{j}$ in the basal plane. This interaction is then rotated into a global coordinate system where the $x$-axis is parallel to $\mathbf{a}^{\star}$, as indicated in figure 3 , giving a dependence of the interaction parameter on the azimuth angle, $\phi_{i j}$, between the projection of $\mathbf{r}_{i}-\mathbf{r}_{j}$ and the $x$-axis.

For each $i$ th hexagonal ion we consider only the couplings $\mathcal{J}^{\mathrm{s}}$ with other hexagonal ions in the same plane, and $\mathcal{J}^{\mathrm{d}}$ with ions in different planes, as shown in figure 3. RPA calculations were performed with the McPhase package [11], and the deduced exchange parameters are summarized in table 1 . We find that the exchange in the basal plane is isotropic $\left(\mathcal{K}^{\mathrm{s} 1}=\right.$ 0 ), whilst that between ions on different planes has a large anisotropic component which is comparable in magnitude to the isotropic component, $\mathcal{J}^{\mathrm{d} 1} \approx \mathcal{K}^{\mathrm{d} 1}$. This reflects the factor 

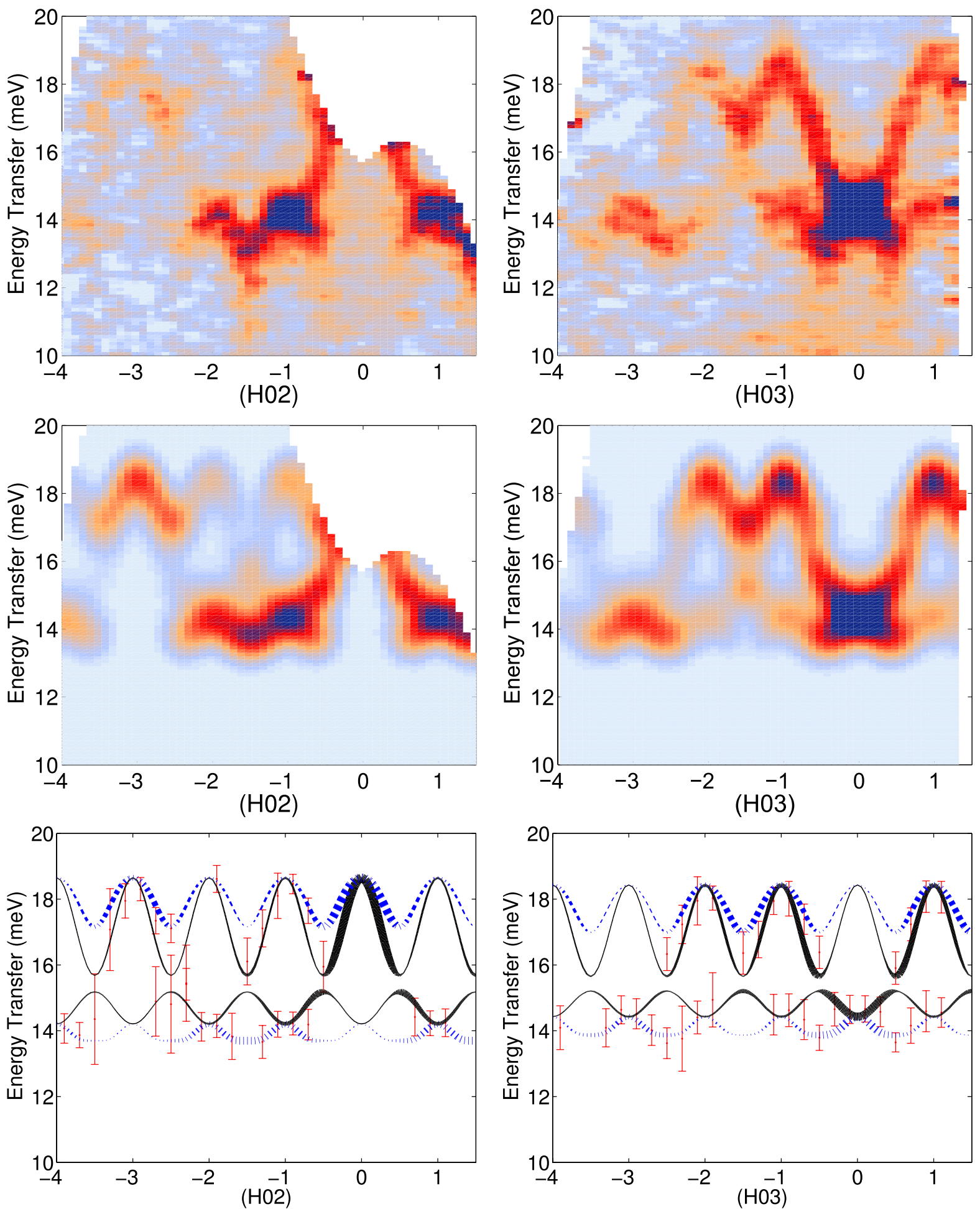

Figure 1. Cuts along $h$ at $l=2$ (left) and $l=3$ (right) showing the dispersion of the hexagonal doublet levels at $3.5 \mathrm{~K}$. The calculated dispersion obtained using the anisotropic exchange interaction model described in the text is shown as a line plot (bottom) and convoluted with a Gaussian lineshape of fixed width (middle). The symmetrized and background subtracted data are shown at the top. Solid black lines show the longitudinal modes, and dashed-dotted blue lines the transverse modes. In general, the two polarizations are mixed, but this mixing vanishes when the excitations, as here, are propagating in the mirror plane $\mathbf{a}^{\star}-\mathbf{c}^{\star}$. The thicknesses of the lines are proportional to the calculated intensities. Red error bars show the centre and half-width at half-maximum of fitted Gaussian peaks to 1D cuts of the data. The cuts were generated at intervals of $h=0.2$ reciprocal lattice units (rlu) integrating over $\Delta h=0.1$ and $\Delta l=0.15$ rlu. The $2 \mathrm{D}$ cuts shown in the upper panels were integrated between $k= \pm 0.1 \mathrm{rlu}$.

of approximately two in the bandwidths of the transverse and longitudinal modes in figure 1 along the $\mathbf{q} \| \mathbf{a}^{\star}$ direction.
Until this point we have considered only the bilinear interactions represented by the operators $\hat{J}_{x}$ and $\hat{J}_{y}$. However, 


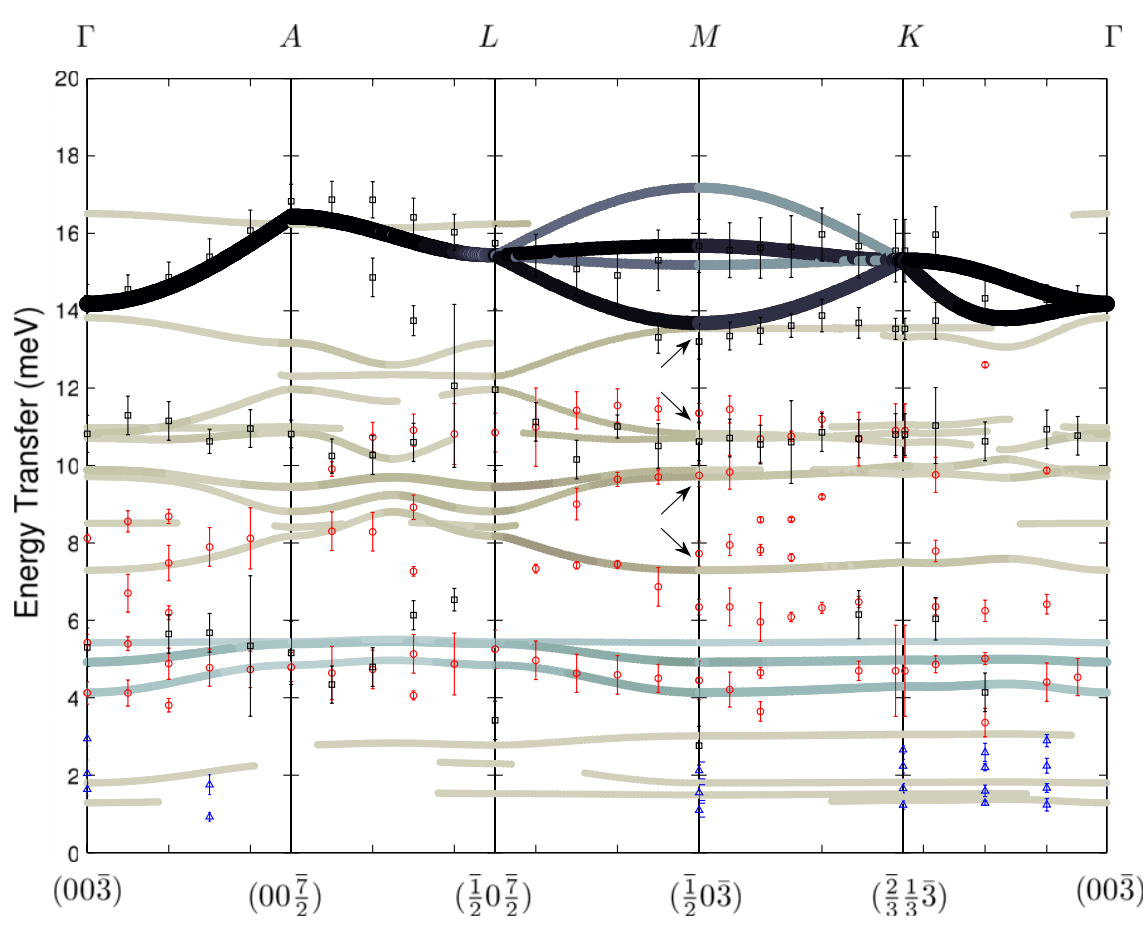

Figure 2. Dispersion in the principal symmetry directions. Red circles are peak centre positions fitted from data taken with $16 \mathrm{meV}$ incident energy, whilst black squares are from $25 \mathrm{meV}$ data. Blue triangles are from [5]. Error bars show the half-width at half-maximum of the peaks. Solid lines indicate the calculated dispersion, with more intense modes in darker shading. The intense branches with energy between 13 and $18 \mathrm{meV}$ arise from transitions to the hexagonal site $\mathrm{CF}$ doublet. Other branches arise from excitations to quasi-cubic $\mathrm{CF}$ levels: the many branches with low intensities above $7 \mathrm{meV}$ are from the doublet at $\approx 10 \mathrm{meV}$, whilst the three branches between 4 and $6 \mathrm{meV}$ are from the singlet. The faint branches below this in energy are due to transitions between the quadrupolar order split ground state doublet. Arrows indicate the four non-degenerate quasi-cubic modes at the M point arising from the $10 \mathrm{meV}$ CF doublet.

Table 1. Deduced exchange parameters in meV. Negative values of $\mathcal{J}$ indicate antiferromagnetic exchange.

\begin{tabular}{lccc}
\hline $\mathcal{J}_{1}^{\mathrm{s} 1}$ & -0.013 & $\mathcal{K}_{1}^{\mathrm{s} 1}$ & 0 \\
$\mathcal{J}_{1}^{\mathrm{d} 1}$ & -0.038 & $\mathcal{K}_{1}^{\mathrm{d} 1}$ & -0.025 \\
$\mathcal{J}_{1}^{\mathrm{d} 2}$ & 0 & $\mathcal{K}_{1}^{\mathrm{d} 2}$ & -0.013 \\
\hline
\end{tabular}

the quadrupolar operators $\hat{Q}_{z x}$ and $\hat{Q}_{y z}$ also have non-zero matrix elements between the singlet and doublet states, and so may be substituted into equations (1) and (2). Equation (3) then becomes

$$
\begin{aligned}
\mathcal{H}= & \sum_{i} \mathcal{H}_{\mathrm{cf}}(i)-\frac{1}{2} \sum_{i j}\left\{\mathcal{J}_{2}^{i j}\left[\hat{Q}_{z x}^{i} \hat{Q}_{z x}^{j}+\hat{Q}_{y z}^{i} \hat{Q}_{y z}^{j}\right]\right. \\
& +\mathcal{K}_{2}^{i j}\left[\cos \left(2 \phi_{i j}\right)\left(\hat{Q}_{z x}^{i} \hat{Q}_{z x}^{j}-\hat{Q}_{y z}^{i} \hat{Q}_{y z}^{j}\right)\right. \\
& \left.\left.+\sin \left(2 \phi_{i j}\right)\left(\hat{Q}_{z x}^{i} \hat{Q}_{y z}^{j}+\hat{Q}_{y z}^{i} \hat{Q}_{z x}^{j}\right)\right]\right\} .
\end{aligned}
$$

As the two types of operators have matrix elements related by $\left\langle s\left|Q_{z x, y z}\right| d\right\rangle=4\left\langle s\left|J_{x, y}\right| d\right\rangle$, if the coupling parameters are scaled such that $\mathcal{J}_{1}^{i j}=4 \mathcal{J}_{2}^{i j}$ and $\mathcal{K}_{1}^{i j}=4 \mathcal{K}_{2}^{i j}$, the exact same calculated spectra are obtained. Thus it is not possible to distinguish which type of interaction is responsible for the dispersive modes from the present data.

From theoretical considerations, an anisotropy of the form (3) could be the result of an anisotropic polarizability of the conduction electrons in an RKKY scenario. Alternatively, the strong quadrupole-quadrupole interactions which drive

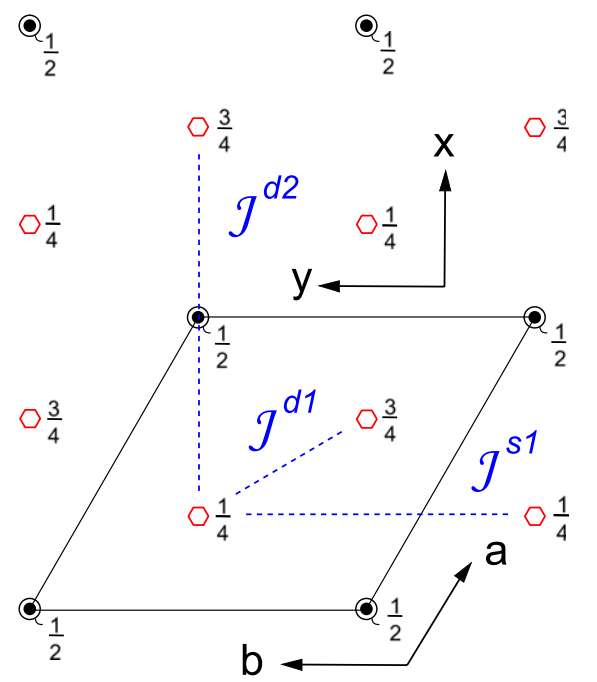

Figure 3. Basal plane projection of the dhcp structure of $\mathrm{UPd}_{3}$ showing only $\mathrm{U}^{4+}$ ions. Quasi-cubic site ions are shown as black circles, hexagonal site ions as red hexagons. Dashed lines show the exchange interaction pathways.

the low temperature ordering of the quasi-cubic sites suggests that the quadrupolar interactions between hexagonal sites may also be significant, leading to anisotropic interactions of the form (4). Such interactions may also arise indirectly from quadrupolar interactions between the hexagonal and quasi-cubic sites. For example, any strain caused by the 
quadrupolar ordering of the quasi-cubic sites can modify the orbital state of the $5 \mathrm{f}$ electrons on the hexagonal sites. This may, in turn, change the charge density of the $5 f$ electrons on the hexagonal sites leading to an electric quadrupole-quadrupole interaction between them.

Experimentally, one might distinguish between a quadrupolar or bilinear interaction by studying the excitations in a magnetic field applied along the basal plane. The antiferromagnetic bilinear interaction would tend to act against the field resulting in a lower Zeeman splitting of the doublet modes than for quadrupolar interactions. We calculate that applying $15 \mathrm{~T}$ along the a-direction would yield a splitting of $\approx 0.1 \mathrm{meV}$ for bilinear interactions, but $\approx 0.3 \mathrm{meV}$ for quadrupolar interactions. The dispersion in a high magnetic field may thus be used to determine the nature of the interactions between the hexagonal sites, although the precision required is, at present, extremely difficult to achieve.

Finally, the dispersion of the quasi-cubic site excitations was calculated [12] using quadrupolar interaction parameters deduced from the bulk properties and XRS measurements described in [2] and shown in figure 2. The low intensity of the data, however, means that there is a large uncertainty in the measured dispersion. Combined with the large number of interaction parameters, it was found to be very difficult to fit both the measured dispersion and bulk properties. Nonetheless the dispersion does support the need for anisotropic quadrupolar interactions between the quasi-cubic sites. In particular, isotropic interactions result in only two modes along LM arising from the crystal field doublet centred around $10 \mathrm{meV}$, whereas the data show evidence of three modes between 6 and $14 \mathrm{meV}$, as do the calculations with anisotropic interactions, which show four modes at the M-point arising from this doublet (denoted by arrows in figure 2). However, along MK the model gives generally flat modes, in contrast to the data which appear to show significant dispersion, especially between 7 and $9 \mathrm{meV}$. There is thus scope for further work, such as including interactions between different quadrupoles on different sites, which the model in [12] neglects.

The calculations were carried out taking into account the ordered quadrupolar structure, and as such show the splitting of the ground state doublet on the quasi-cubic sites into four modes, which reasonably accounts for the measurements of McEwen et al [5] shown as blue triangles in figure 2 .

In conclusion, we have measured the dispersive crystal field excitations of $\mathrm{UPd}_{3}$ using inelastic neutron scattering, and determined that anisotropic two-ion interactions are needed to describe the data. We have verified that the quadrupolar interaction parameters for the quasi-cubic sites deduced from XRS and bulk properties measurements are valid, and obtained exchange parameters for the hexagonal site interactions.

\section{Acknowledgment}

We thank R A Ewings for assistance with the Horace program.

\section{References}

[1] Jensen J and Mackintosh A R 1991 Rare Earth Magnetism (Oxford: Clarendon) http://www.fys.ku.dk/ jjensen/REM. htm

[2] Walker H C, McEwen K A, Le M D, Paolasini L and Fort D 2008 J. Phys.: Condens. Matter 20395221

[3] Bewley R I, Eccleston R S, McEwen K A, Hayden S M, Dove M T, Bennington S M, Treadgold J R and Coleman R L S 2006 Physica B 385/386 1029-31

[4] Perring T G, Ewings R A and Duijn J V 2010 http://horace. isis.rl.ac.uk

[5] McEwen K A, Steigenberger U and Martinez J L 1993 Physica B 186-188 670-4

[6] McEwen K A, Park J G, Gipson A J and Gehring G A 2003 J. Phys.: Condens. Matter 15 S1923-35

[7] McEwen K A, Walker H C, Le M D, McMorrow D F, Colineau E, Wastin F, Wilkins S B, Park J G, Bewley R I and Fort D 2007 J. Magn. Magn. Mater. $310718-22$

[8] Le M D 2009 Magnetism and quadrupolar order in f-electron systems PhD Thesis University College London

[9] Buyers W J L, Murray A F, Holden T M, Svensson E C, de V DuPlessis P, Lander G H and Vogt O 1980 Physica $\mathrm{B}+\mathrm{C} 102$ 291-8

[10] Houmann J G, Rainford B D, Jensen J and Mackintosh A R 1979 Phys. Rev. B 20 1105-18

[11] Rotter M 2006 Comput. Mater. Sci. 38 400-4

[12] Le M D, McEwen K A, Rotter M, Doerr M, Barcza A, Park J G, Brooks J, Jobiliong E and Fort D 2011 in preparation 